\title{
Exciton localization and decomposition dynamics in cuprous halide nanocrystals
}

\author{
Tsuyoshi Okuno, Hiroshi Miyajima, Akihiro Satake, and Yasuaki Masumoto \\ Institute of Physics and Center for TARA (Tsukuba Advanced Research Alliance), University of Tsukuba, Tsukuba, Ibaraki 305, Japan
}

(Received 10 July 1996)

\begin{abstract}
We report temporal changes of luminescence and absorption (differential transmission) of excitons in nanometer-size semiconductor crystals (nanocrystals) of $\mathrm{CuCl}$ embedded in $\mathrm{NaCl}$ or in glass, and $\mathrm{CuBr}$ nanocrystals embedded in glass. In $\mathrm{CuCl}$ nanocrystals in $\mathrm{NaCl}$, an exciton relaxes nonradiatively to some localized state. In $\mathrm{CuCl}$ and $\mathrm{CuBr}$ nanocrystals in glass, the temporal changes of the differential transmission have a longer decay component in addition to the fast decay component which agrees with the luminescence decay. This result suggests exciton decomposition and the existence of an electron or a hole remainder in the nanocrystals. The decay time of the longer decay component increases by the accumulation of photoexcitation. This phenomenon indicates persistent trapping of carriers in the glass matrix, which is concerned with persistent spectral hole burning in nanocrystals. [S0163-1829(96)11449-1]
\end{abstract}

\section{INTRODUCTION}

In this decade, semiconductor nanometer-size microcrystallites (nanocrystals) have attracted much attention even since the quantum size effect was observed in them. ${ }^{1-4}$ One of the interests in nanocrystals is motivated by theoretical studies of the enhancement of the oscillator strength and the third-order susceptibility for confined excitonic systems. ${ }^{5,6} \mathrm{~A}$ super-radiant decay, in the sense that a coherently superposed polarization is involved, was reported in Wannier excitons in $\mathrm{CuCl}$ nanocrystals. 7,8

In the studies mentioned above the optical properties of I-VII nanocrystals were characterized only by the quantum confinement, and little attention seemed to be paid to the effect of the surface or the interface of nanocrystals embedded in crystals or glasses. In II-VI nanocrystals which show large Stokes-shifted exciton luminescence, the importance of surface states was recognized and studied. ${ }^{9}$ In another paper on II-VI nanocrystals, photoionization of nanocrystals is discussed. ${ }^{10}$ In addition, nanocrystalline systems including I-VII nanocrystals widely reveal persistent spectral hole burning, and this phenomenon suggests electron or hole trapping at the surface or in the matrix (photoionization of nanocrystals). ${ }^{11-13}$ Therefore, it is possible that the relaxation of excitons in I-VII nanocrystals is affected by the surface or matrix states. In other words, carrier transfer to the surface or the matrix may affect the relaxation mechanism of excitons.

As for the systems in which charge transfer takes place in real space, type-II quantum wells are known. In type-II $(\mathrm{Al}, \mathrm{Ga}) \mathrm{As} / \mathrm{Al}$ As quantum wells, the real-space $\Gamma-X$ transfer of electrons determines the lifetime of luminescence of $\Gamma-\Gamma$ heavy-hole excitons, since the lowest conduction-band state is in the $X$ minima of the AlAs layers. In these systems, the luminescence lifetime of $\Gamma-\Gamma$ excitons is reported to be in an order of $100 \mathrm{fs} \sim 10 \mathrm{ps},{ }^{14,15}$ this time corresponds to the transfer-time of photoexcited electrons from the $\Gamma$ state in well layers to the $X$ state in AlAs layers. However, temporal changes of induced absorption show not only the similar fast decay (100 fs-10 ps) but also much slower tail. ${ }^{15,16}$ This slower component mainly reflects annihilation of holes remaining in the $\Gamma$ valence state in well layers.
In order to investigate carrier dynamics in intrinsic semiconductors, time-resolved measurements of luminescence as well as induced absorption are useful. Exciton luminescence is proportional to the number of pairs of electrons and holes, while the sum of electron number and hole number is reflected in induced absorption. Since numbers of electrons and holes are equal to each other in ordinary cases of intrinsic semiconductors, temporal change of the luminescence intensity and that of the induced absorption intensity agree with each other. Nevertheless, in such cases as type-II quantum wells where electrons and holes are decomposed into different layers, temporal changes of luminescence and induced absorption can be different from each other. Measurements of both experiments and comparison of them are useful and important to discuss carrier dynamics.

In this paper, we report the experimental study of temporal changes of luminescence and differential transmission of Wannier excitons in $\mathrm{CuCl}$ nanocrystals embedded in $\mathrm{NaCl}$ crystals, and $\mathrm{CuCl}$ or $\mathrm{CuBr}$ nanocrystals embedded in glasses. From these results, we clarify an important role of "localized" or trap states on the relaxation mechanisms of excitons. Then, we discuss the relation between the relaxation mechanism and persistent hole-burning phenomena.

\section{EXPERIMENTAL PROCEDURE}

The samples under investigation are $\mathrm{CuCl}$ nanocrystals embedded in $\mathrm{NaCl}$ crystals, ${ }^{17,18} \mathrm{CuCl}$ nanocrystals embedded in glass, and $\mathrm{CuBr}$ nanocrystals embedded in glass (potassium aluminoborosilicate glass was used for the glass matrix). These nanocrystals were grown by heat treatment. The mean sizes of the nanocrystals were measured by small-angle $\mathrm{x}$-ray scattering and were $2.5 \mathrm{~nm}$ for $\mathrm{CuCl}$ nanocrystals in $\mathrm{NaCl}, 3.5 \mathrm{~nm}$ for $\mathrm{CuCl}$ nanocrystals in glass, and $3.6 \mathrm{~nm}$ for $\mathrm{CuBr}$ nanocrystals in glass. The samples were directly immersed in superfluid helium at $2 \mathrm{~K}$ in an optical cryostat. In order to measure absorption spectra, a halogen lamp was used. As an exciting source for measurement of luminescence, frequency-doubled 100-fs pulses from a mode-locked Ti:sapphire laser were used. The excitation photon energy was $3.37 \mathrm{eV}$. Temporal changes of luminescence were mea- 

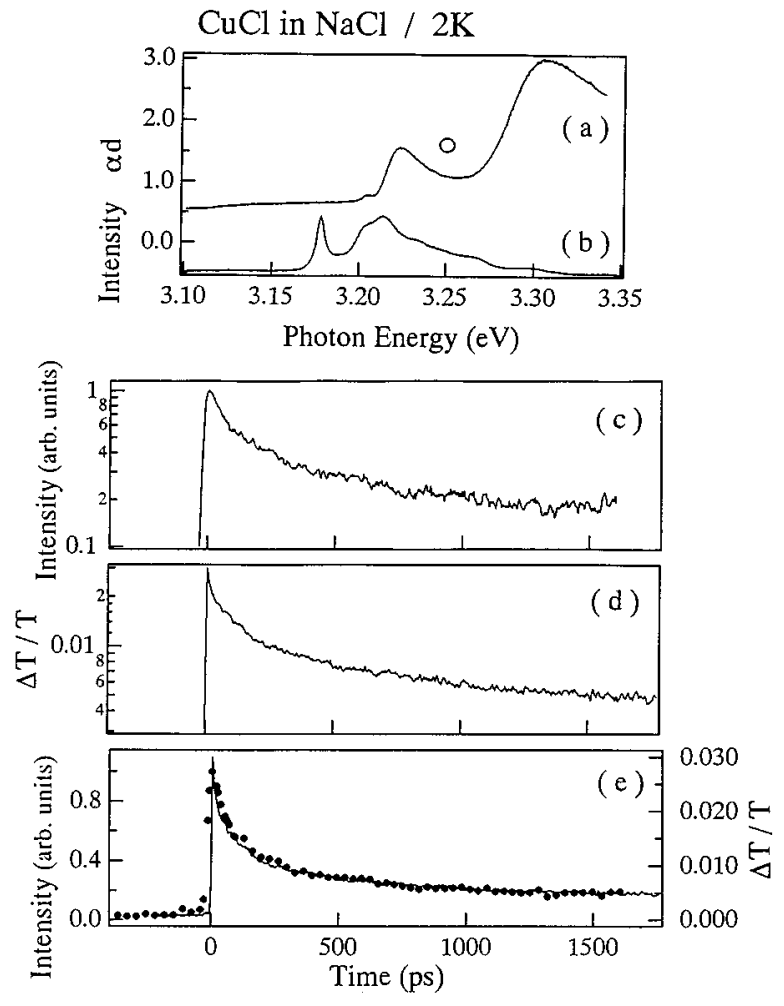

FIG. 1. (a) Absorption spectrum of $\mathrm{CuCl}$ nanocrystals embedded in $\mathrm{NaCl}$ crystal measured at $2 \mathrm{~K}$. (b) Luminescence spectrum with an excitation photon energy of $3.37 \mathrm{eV}$. (c) Temporal change of luminescence at $3.25 \mathrm{eV}$ shown on a log scale. The excitation density is $0.1 \mu \mathrm{J} / \mathrm{cm}^{2}$. (d) Temporal change of differential transmission at $3.25 \mathrm{eV}$ shown on a log scale. The excitation density is $20 \mu \mathrm{J} / \mathrm{cm}^{2}$. (e) Temporal changes of luminescence (circles) and differential transmission (line) shown on a linear scale.

sured by using a synchroscan streak camera. Time resolution of this measurement was about $15 \mathrm{ps}$. In order to obtain temporal changes of differential transmission by pump-probe measurements, frequency-doubled pulses with a repetition rate of $250 \mathrm{kHz}$ from a regenerative amplifier of the Ti:sapphire laser were used. A laser beam was split into two; one is used as a pump beam, and the other with the intensity of $\sim 1 / 10$ of the pump beam was used as a probe beam. Time resolution of this pump-probe measurement was about 300 fs.

\section{RESULTS AND DISCUSSION}

\section{A. Comparison of temporal changes between luminescence and differential transmission}

Figure 1(a) shows the absorption spectrum of $\mathrm{CuCl}$ nanocrystals embedded in $\mathrm{NaCl}$ obtained at $2 \mathrm{~K}$. The $Z_{3}$ and $Z_{1,2}$ exciton peaks are located around $3.22 \mathrm{eV}$ and $3.30 \mathrm{eV}$, respectively. Figure 1(b) shows the luminescence spectrum. This luminescence can be roughly separated into two parts; one peak is located at $3.175 \mathrm{eV}$ and the other broader luminescence band with several structures is around $3.21 \mathrm{eV}$. The peak at $3.175 \mathrm{eV}$ is attributed to an exciton bound to a neutral acceptor $I_{1},{ }^{19}$ and we do not discuss this peak in this paper. The other luminescence band around $3.21 \mathrm{eV}$ corresponds to the $Z_{3}$ exciton. When we compare the lumines-

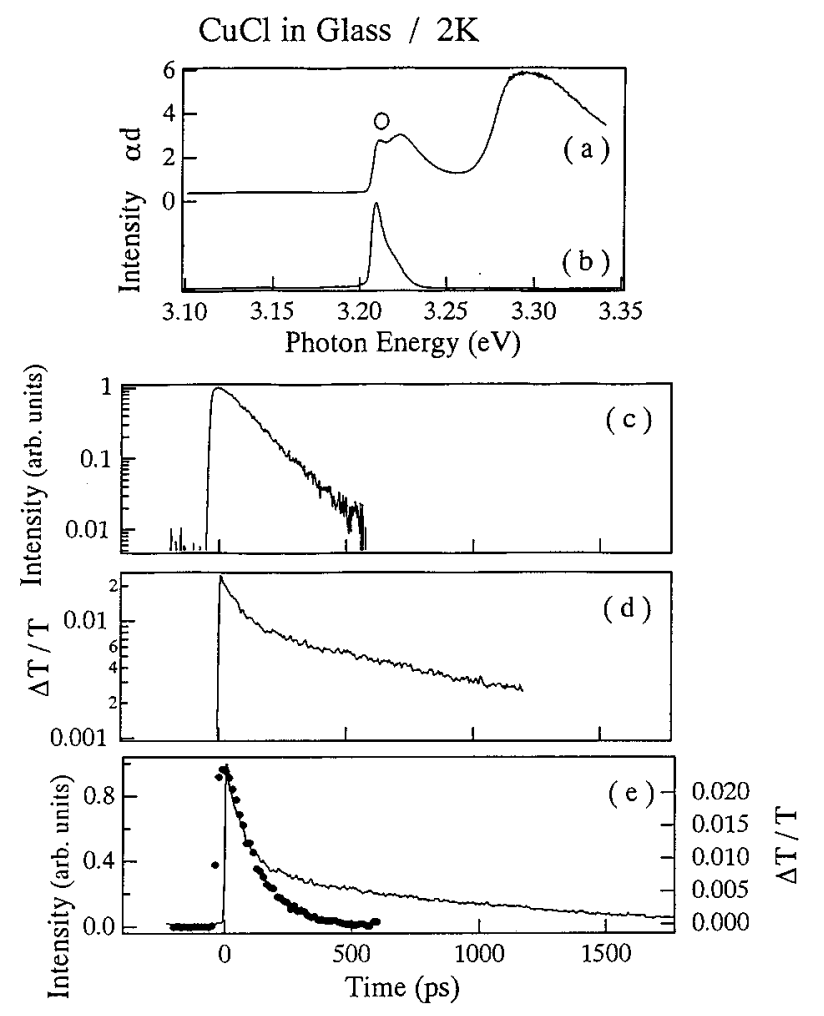

FIG. 2. (a) Absorption spectrum of $\mathrm{CuCl}$ nanocrystals embedded in glass measured at $2 \mathrm{~K}$. (b) Luminescence spectrum with an excitation photon energy of $3.37 \mathrm{eV}$. (c) Temporal change of luminescence at $3.21 \mathrm{eV}$ shown on a log scale. The excitation density is $0.004 \mu \mathrm{J} / \mathrm{cm}^{2}$. (d) Temporal change of differential transmission at $3.21 \mathrm{eV}$ shown on a $\log$ scale. The excitation density is 0.4 $\mu \mathrm{J} / \mathrm{cm}^{2}$. (e) Temporal changes of luminescence (circles) and differential transmission (line) shown on a linear scale.

cence peak with the absorption peak of the $Z_{3}$ exciton, we find that this luminescence shows Stokes shift of $\sim 12 \mathrm{meV}$. Thus the dominant luminescence is thought to come from some "localized" state. ${ }^{20}$ In order to avoid the contribution of the "localized" state to the luminescence and to discuss the dynamics of "free" $Z_{3}$ exciton, we made experiments of time-resolved luminescence and pump-probe measurements at $3.25 \mathrm{eV}$ denoted by the circle in Fig. 1(a).

Figure 1(c) shows the semilogarithmic plot of the temporal change of the luminescence observed at $3.25 \mathrm{eV}$; the excitation density was $0.1 \mu \mathrm{J} / \mathrm{cm}^{2}$. We find that this luminescence decay cannot be fitted by single-exponential decay but by two exponential decay; two decay time constants are approximately $100 \mathrm{ps}$ and 2 ns. Figure 1 (d) shows the temporal change of differential transmission at $3.25 \mathrm{eV}$ plotted on a log scale under the excitation density of $20 \mu \mathrm{J} / \mathrm{cm}^{2}$. This profile also shows two-exponential decay with decay time constants of $\sim 100 \mathrm{ps}$ and $\sim 2 \mathrm{~ns}$, and is quite similar to the luminescence-decay profile in Fig. 1(c). In Fig. 1(e), both of the temporal changes in Figs. 1(c) and 1(d) are plotted on a linear scale; circles are the results of the luminescence and the line is of the differential transmission. We find that both data are in good agreement. Slight difference of rise times around 0 ps between two data comes from the difference of the time resolution between the two experiments.

Figure 2 shows results for $\mathrm{CuCl}$ nanocrystals in glass. 
Figure 2(a) shows the absorption spectrum and Fig. 2(b) shows the luminescence spectrum. We find that the luminescence band is overlapped with the absorption band of the $Z_{3}$ exciton. Disagreement between the luminescence peak and the absorption peak may come from smaller luminescent efficiency of smaller nanocrystals with larger absorption photon energy. Figure 2(c) shows the temporal change of the luminescence observed at the luminescence-peak energy of $3.21 \mathrm{eV}$ denoted by the circle in Fig. 2(a); the excitation density was $0.004 \mu \mathrm{J} / \mathrm{cm}^{2}$. We find that this luminescence decay is well fitted by the single-exponential decay; the decay time constant is approximately $100 \mathrm{ps}$.

Figure 2(d) shows the temporal change of differential transmission at $3.21 \mathrm{eV}$ under the excitation density of 0.4 $\mu \mathrm{J} / \mathrm{cm}^{2}$. Although the temporal changes of luminescence and differential transmission of $\mathrm{CuCl}$ nanocrystals in $\mathrm{NaCl}$ in Fig. 1(e) are quite similar to each other, the profiles of $\mathrm{CuCl}$ nanocrystals in glass in Figs. 2(c) and 2(d) are different. Even if we use the same excitation density in both experiments, this disagreement remains. The profile of differential transmission in Fig. 2(d) cannot be fitted by singleexponential decay, but by two-exponential decay; the fast decay time constant is $\sim 100 \mathrm{ps}$, which agrees with the decay time constant of luminescence in Fig. 2(c), and the slow decay time constant is $\sim 1$ ns. In Fig. 2(e), both of the temporal changes in Figs. 2(c) and 2(d) are plotted together. From 0 ps to 100 ps two data agree well with each other. Then the luminescence (circles) decays in a singleexponential profile, while the differential transmission (line) has a longer decay component.

The results of $\mathrm{CuBr}$ nanocrystals in glass shown in Fig. 3 are similar to the results of $\mathrm{CuCl}$ nanocrystals in glass. Figure 3(a) shows the absorption spectrum, and the $Z_{1,2}$ absorption band is located around $3.07 \mathrm{eV}$. Figure 3(b) shows the luminescence spectrum. The luminescence band is at the lower-energy tail of the absorption band.

Figure 3(c) shows the temporal change of the luminescence observed at the luminescence-peak energy of $3.02 \mathrm{eV}$ denoted by the circle in Fig. 3(a); the excitation density was $0.1 \mu \mathrm{J} / \mathrm{cm}^{2}$. We find that this luminescence decay is well fitted by single-exponential decay with the decay time constant of $\sim 20$ ps. Figure 3(d) shows the temporal change of differential transmission at $3.02 \mathrm{eV}$ under the excitation density of $4 \mu \mathrm{J} / \mathrm{cm}^{2}$. This profile is different from that in Fig. $3(\mathrm{c})$, and is fitted by two-exponential decay; the decay time constants are $\sim 20$ ps and $\sim 70$ ps. In Fig. 3(e), both of the temporal changes in Figs. 3(c) and 3(d) are plotted. From 0 ps to $10 \mathrm{ps}$ two data agree well with each other. Then the luminescence (circles) decays in a single-exponential profile, while the differential transmission (line) has a longer decay component.

\section{B. Relaxation mechanism of excitons}

In $\mathrm{CuCl}$ nanocrystals in $\mathrm{NaCl}$, the $Z_{3}$ exciton relaxes in the decay time constants of $\sim 100$ ps and $\sim 2$ ns. As will be discussed in the forthcoming paper, luminescence rise is observed at the lower energy $(\sim 3.20 \mathrm{eV})$ region of the "localized" state; the rise time agrees with the fast decay time (in this case, $\sim 100 \mathrm{ps}$ ) in the high-energy region of the "free" $Z_{3}$ exciton. Therefore, the fast decay component of $\sim 100 \mathrm{ps}$
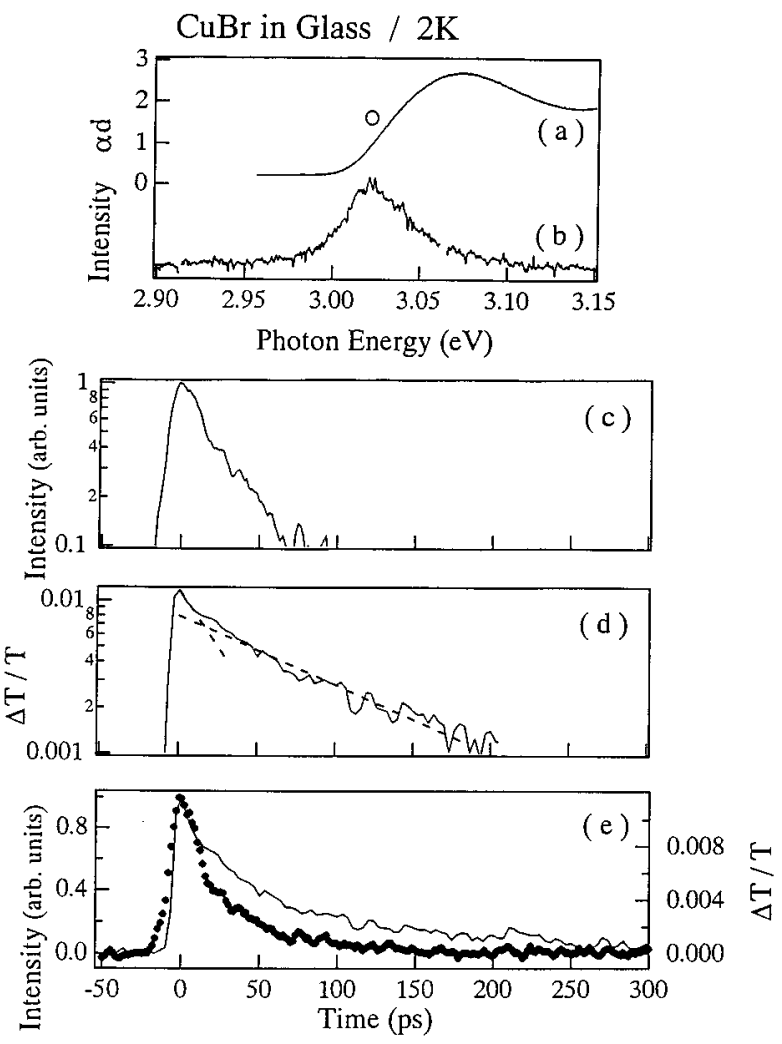

FIG. 3. (a) Absorption spectrum of $\mathrm{CuBr}$ nanocrystals embedded in glass measured at $2 \mathrm{~K}$. (b) Luminescence spectrum with the excitation photon energy of $3.37 \mathrm{eV}$. (c) Temporal change of luminescence at $3.02 \mathrm{eV}$ shown on a log scale. The excitation density is $0.1 \mu \mathrm{J} / \mathrm{cm}^{2}$. (d) Temporal change of differential transmission at $3.02 \mathrm{eV}$ shown on a $\log$ scale. The excitation density is 4 $\mu \mathrm{J} / \mathrm{cm}^{2}$. (e) Temporal changes of luminescence (circles) and differential transmission (line) shown on a linear scale.

is thought to be nonradiative relaxation time from the "free" $Z_{3}$ exciton to the "localized" state (the detailed experimental results and discussion will be shown in the subsequent paper). The appearance of the longer decay component means that some nanocrystals have no "localized" state to which "free" $Z_{3}$ excitons will relax. We assume that this time constant of $\sim 2$ ns corresponds to the radiative recombination time of the exciton. The exciton relaxation dynamics in $\mathrm{CuCl}$ nanocrystals in $\mathrm{NaCl}$ mentioned above is depicted in Fig. 4(a).

In $\mathrm{CuCl}(\mathrm{CuBr})$ nanocrystals in glass, the temporal change of luminescence does not agree with that of differential transmission. This result can be understood by nonradiative decomposition of excitons as is depicted in Fig. 4(b). In some nanocrystals, a photoexcited exciton is decomposed nonradiatively into an electron and a hole. They may be trapped into defects or surfaces. We assume that an electron or a hole remains in the nanocrystal and that an electron-hole pair recombine nonradiatively in the time constant of $\sim 1 \mathrm{~ns}$ in $\mathrm{CuCl}$ nanocrystals in glass $(\sim 70 \mathrm{ps}$ in $\mathrm{CuBr}$ nanocrystals in glass). Then these nanocrystals contribute to the longer decay time constant of $\sim 1 \mathrm{~ns}(\sim 70 \mathrm{ps})$ of the differential transmission, but not to luminescence. In other nanocrystals, excitons relax radiatively or nonradiatively in the decay time constant of $\sim 100 \mathrm{ps}(\sim 20 \mathrm{ps})$, which is reflected in the fast 
(a) $\mathrm{CuCl}$ in $\mathrm{NaCl}$

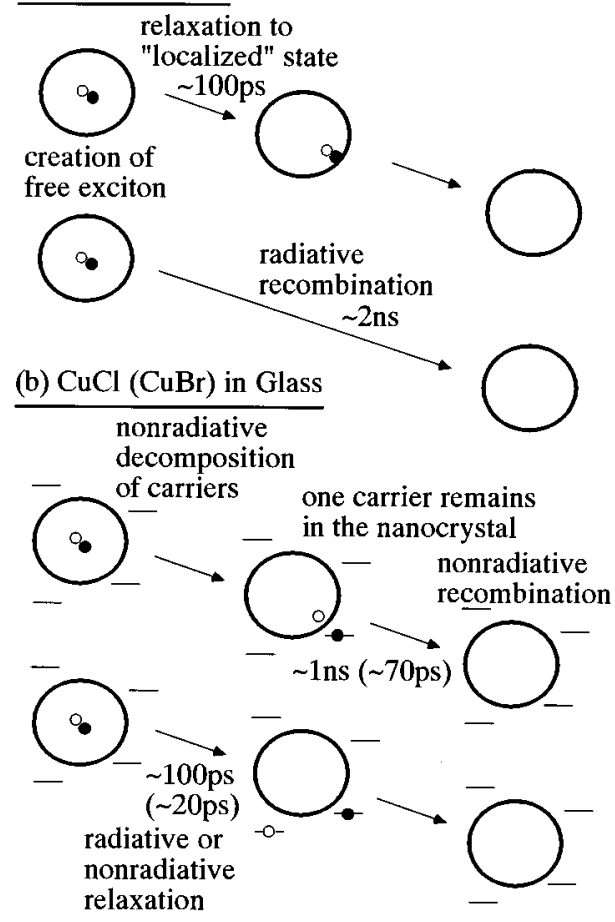

FIG. 4. Schematic drawing of relaxation mechanisms of excitons in $\mathrm{CuCl}$ nanocrystals embedded in $\mathrm{NaCl}$ crystal, and in $\mathrm{CuCl}$ (or $\mathrm{CuBr}$ ) nanocrystals embedded in glass.

decay component of differential transmission and in the single-exponential decay of luminescence. In this way, we can explain the disagreement between the temporal changes of the luminescence and the differential transmission.

In our previous paper, we reported persistent spectral hole burning in $\mathrm{CuBr}$ nanocrystals in glass. ${ }^{13}$ This phenomenon was ascribed to electron trapping at the surface of the nanocrystals and hole tunneling into traps in the glass host. Following this consideration, the fast decay time constant of $\sim 100 \mathrm{ps}(\sim 20 \mathrm{ps})$ corresponds to the trapping time of electrons and the tunneling time of holes. The long decay time constant of $\sim 1 \mathrm{~ns}(\sim 70 \mathrm{ps})$ corresponds to the nonradiative recombination time between the electron at the surface and the hole at the traps in the glass. At this time, some of these carriers remain without recombination, and induce persistent spectral hole burning.

In $\mathrm{CuCl}$ nanocrystals in $\mathrm{NaCl}$, the temporal changes of luminescence and differential transmission agree with each other, and thus we need not assume decomposition of excitons. Considering that the band gap of $\mathrm{NaCl}$ is $8.6 \mathrm{eV}$ (Ref. 21) and the optical gap of the glass is $4.7 \mathrm{eV}$, barrier height for the carriers in $\mathrm{CuCl}$ nanocrystals in $\mathrm{NaCl}$ is higher than that in the glass matrix. This is thought to be the reason why decomposition of excitons scarcely takes place in $\mathrm{CuCl}$ nanocrystals in $\mathrm{NaCl}$. However, some excitons in $\mathrm{CuCl}$ nanocrystals in $\mathrm{NaCl}$ may be decomposed and electrons or holes may be trapped at the surface or in the matrix, which induces persistent spectral hole burning in $\mathrm{CuCl}$ nanocrystals in $\mathrm{NaCl}$. The hole-burning quantum efficiency of $\mathrm{CuCl}$ nanocrystals in $\mathrm{NaCl}$ is smaller than that of $\mathrm{CuCl}$ nanocrystals in glass, ${ }^{22}$ and this observation is consistent with the (a) $\mathrm{CuCl}$ in glass $(2 \mathrm{~K})$

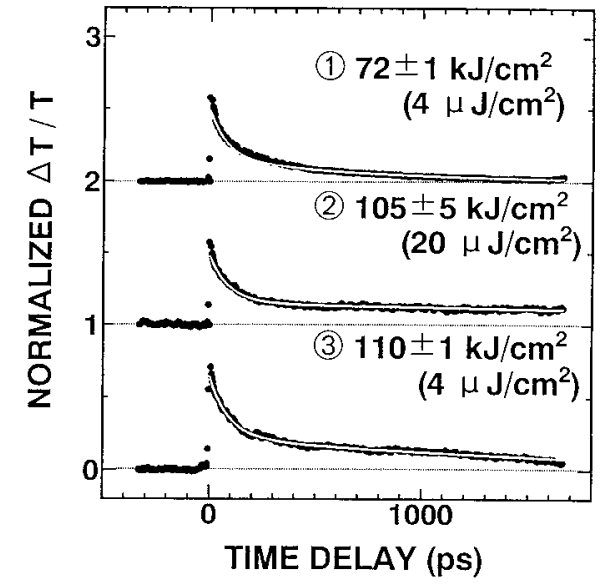

(b) CuBr in glass (2 K)

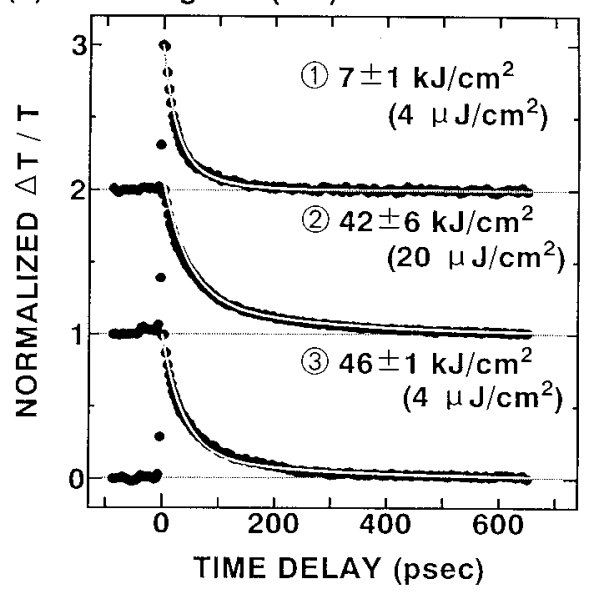

FIG. 5. Photoaccumulation effects on the temporal changes of differential transmission in (a) $\mathrm{CuCl}$ or (b) $\mathrm{CuBr}$ nanocrystals embedded in glass. Three temporal changes drawn by symbols are measured successively from the top to the bottom. The accumulated excitation densities (and the pump-beam densities in the parentheses) are shown in the figure. The excitation photon energy was (a) $3.22 \mathrm{eV}$ or (b) $3.09 \mathrm{eV}$. Lines are results of fitting by the twoexponential decay of $I_{0}\left[a \exp \left(-t / \tau_{a}\right)+b \exp \left(-t / \tau_{b}\right)\right]$.

different decomposition probability between excitons in the nanocrystals in glass and those in the nanocrystals in $\mathrm{NaCl}$.

\section{Photoaccumulation effect on the transmission time trace}

In the temporal changes of differential transmission in $\mathrm{CuCl}$ or $\mathrm{CuBr}$ nanocrystals in glass, we noticed the photoaccumulation effect. Figure 5 shows the results of (a) $\mathrm{CuCl}$ and (b) $\mathrm{CuBr}$ nanocrystals in glass. In both the samples, three time traces drawn by symbols were obtained successively from the top to the bottom. For $\mathrm{CuCl}$ nanocrystals in glass, the accumulated excitation densities and the pumpbeam energy densities in the parentheses were shown in Fig. $5(\mathrm{a})$, and the excitation photon energy was $3.22 \mathrm{eV}$. For $\mathrm{CuBr}$ nanocrystals in glass, the accumulated excitation densities and the pump-beam densities were shown in Fig. 5(b), and the excitation photon-energy was $3.09 \mathrm{eV}$. The results shown in Figs. 2(d) and 3(d) correspond to the top traces in Fig. 5 obtained at the smallest accumulated intensities. All the temporal changes in Fig. 5 can be fitted by two- 
exponential decay, given by $I_{0}$ $\left[a \exp \left(-t / \tau_{a}\right)+b \exp \left(-t / \tau_{b}\right)\right]$. The used parameters for the fitting in Fig. 5(a) are (1) $I_{0}=1, a=0.64, \tau_{a}=71 \mathrm{ps,}$ $b=0.35, \tau_{b}=0.67 \mathrm{~ns}$, (2) $I_{0}=1.2, a=0.75, \tau_{a}=71 \mathrm{ps,}$ $b=0.25, \tau_{b}=6.2 \mathrm{~ns}$, and (3) $I_{0}=0.48, a=0.70, \tau_{a}=74 \mathrm{ps}$, $b=0.30, \tau_{b}=2$ ns. Here, $I_{0}$ in (1) was set to be unity, and $I_{0}$ in (2) and (3) represents the relative intensity. In the same manner, the used parameters in Fig. 5(b) are (1) $I_{0}=1$, $a=0.71, \tau_{a}=22 \mathrm{ps}, \quad b=0.29, \tau_{b}=67 \mathrm{ps}$, (2) $I_{0}=29$, $a=0.86, \tau_{a}=43 \mathrm{ps}, b=0.24, \tau_{b}=250 \mathrm{ps}$, and (3) $I_{0}=0.52$, $a=0.85, \tau_{a}=38 \mathrm{ps}, b=0.15, \tau_{b}=280 \mathrm{ps}$.

When we compare the trace (1) with the trace (3), we can find that the fast decay time constant is about the same but the longer decay time constant increases. These traces (1) and (3) were obtained by the same pump density but a different accumulated density. If we compare the time integral of trace (1) with that of trace (3), we find that the integral was scarcely changed. We can say that the photoaccumulation effect means the increase of the long decay time constant which was ascribed to the nonradiative recombination time discussed in the previous section.

The increase of the long decay time constant can be explained by the following scenario concerned with persistent spectral hole burning. A photoexcited exciton is decomposed, and then the electron is captured at the surface and the hole is at the trap in the glass. At the small accumulated densities, the hole is captured by a trap near the photoexcited nanocrystal. At this time, an exciton is easily decomposed in a nanocrystal when a trap is located near the nanocrystal. In this case, the electron and the hole recombine quickly $(\sim 1$ ns for $\mathrm{CuCl}$ nanocrystals in glass, or $\sim 70$ ps for $\mathrm{CuBr}$ nanocrystals in glass), and the small portion of the pairs are left without recombination. These pairs induce persistent spectral hole burning. With the increase of the accumulated density of photoexcitation, traps near nanocrystals capture holes and become inactive. Then a hole coming from the decomposed exciton is captured in traps distant from the nanocrystals. The recombination time of a far separated electron-hole pair becomes longer. In this way, we can explain the increase of the long decay time constant in the transmission time trace. As for the relationship between this photoaccumulation effect and persistent spectral holeburning phenomena, we note that a persistent hole is found in an absorption spectrum after the measurement of Fig. 5.

In contrast with the differential transmission, no photoaccumulation effect was observed on luminescence. The reason may be that separated electron-hole pairs do not luminesce. Temporal changes of luminescence show singleexponential profiles without a longer decay component coming from separated pairs. Hence only electron-hole pairs without separation (excitons) are thought to contribute to luminescence. When a photoexcited exciton is decomposed, the electron-hole pair recombines nonradiatively (without luminescence) or is left without recombination. Thus some of the separated pairs without recombination induce photoaccumulation effect on the differential transmission but not on luminescence.

We did not observe the photoaccumulation effect on the transmission time trace in $\mathrm{CuCl}$ nanocrystals in $\mathrm{NaCl}$ so far. In this system, the long decay time constant ( $\sim 2 \mathrm{~ns})$ was not ascribed to the recombination time of a separated and trapped electron-hole pair but to the radiative recombination time of the exciton. Thus the long decay component has no relation to the persistent spectral hole burning and the photoaccumulation effect. Further experimental study is necessary to clarify the exciton dynamics causing hole-burning phenomena in $\mathrm{CuCl}$ nanocrystals in $\mathrm{NaCl}$, because we did not find any photoaccumulation effect on the exciton dynamics.

\section{CONCLUSION}

We measured temporal changes of luminescence and absorption of excitons in $\mathrm{CuCl}$ nanocrystals embedded in $\mathrm{NaCl}$ or glass, and $\mathrm{CuBr}$ nanocrystals embedded in glass. Both of the temporal changes for $\mathrm{CuCl}$ nanocrystals in $\mathrm{NaCl}$ agree well with each other, and show two-exponential decay. Two decay time constants of $\sim 100$ ps and $\sim 2$ ns correspond to relaxation to the "localized" state and radiative recombination, respectively. In $\mathrm{CuCl}$ and $\mathrm{CuBr}$ nanocrystals in glass, the temporal changes of the differential transmission show two-exponential decay, and are different from the singleexponential luminescence decay; the decay time constant of the luminescence agrees with the fast decay time constant in the differential transmission. This disagreement between two decay profiles shows nonradiative decomposition of excitons and existence of an electron or a hole remainder in the nanocrystals. In addition, in the temporal changes of the differential transmission in $\mathrm{CuCl}$ or $\mathrm{CuBr}$ nanocrystals in glass, the decay time constant of the longer decay component increases with the increase of accumulated excitation density. This result shows persistent trapping of carriers, which is thought to be the origin of persistent spectral hole-burning phenomena in nanocrystals.

\section{ACKNOWLEDGMENTS}

Small-angle $\mathrm{X}$-ray-scattering experiments were done at the Photon Factory (PF) of the National Laboratory for High Energy Physics by the approval of the PF Advisory Committee (Proposals Nos. 92-117). The authors wish to thank Professor Y. Amemiya in PF for his guidance to the small-angle $\mathrm{X}$-ray-scattering experiments. The authors would like to express appreciation for Dr. Y. Yamada's collaboration at the early stage of this study.

\footnotetext{
${ }^{1}$ A. I. Ekimov, A. L. Efros, and A. A. Onushchenko, Solid State Commun. 56, 921 (1985).

${ }^{2}$ L. E. Brus, J. Chem. Phys. 80, 4403 (1984).

${ }^{3}$ A. D. Yoffe, Adv. Phys. 42, 173 (1993).
}

\footnotetext{
${ }^{4}$ L. Bányai and S. W. Koch, Semiconductor Quantum Dot (World Scientific, Singapore, 1993).

${ }^{5}$ E. Hanamura, Phys. Rev. B 38, 1228 (1988).

${ }^{6}$ Y. Kayanuma, Phys. Rev. B 38, 9797 (1988).
} 
${ }^{7}$ A. Nakamura, H. Yamada, and T. Tokizaki, Phys. Rev. B 40, 8585 (1989).

${ }^{8}$ T. Itoh, M. Furumiya, T. Ikehara, and C. Gourdon, Solid State Commun. 73, 271 (1990).

${ }^{9}$ M. G. Bawendi, W. L. Wilson, L. Rothberg, P. J. Carroll, T. M. Jedju, M. L. Steigerwald, and L. E. Brus, Phys. Rev. Lett. 65, 1623 (1990).

${ }^{10}$ D. I. Chepic, Al. L. Efros, A. I. Ekimov, M. G. Ivanov, V. A. Kharchenko, I. A. Kudriavtsev, and T. V. Yazeva, J. Lumin. 47, 113 (1990).

${ }^{11}$ Y. Masumoto, L. G. Zimin, K. Naoe, S. Okamoto, and T. Arai, Mater. Sci. Eng. B 27, L5 (1994).

${ }^{12}$ K. Naoe, L. G. Zimin, and Y. Masumoto, Phys. Rev. B 50, 18200 (1994).

${ }^{13}$ Yasuaki Masumoto, Tadashi Kawazoe, and Tetsuya Yamamoto, Phys. Rev. B 52, 4688 (1995).

${ }^{14}$ G. Peter, E. Göbel, W. W. Ruhle, J. Nagle, and K. Ploog, Superlatt. Microstruct. 5, 197 (1989).
${ }^{15}$ J. Feldmann, J. Nunnenkamp, G. Peter, E. Göbel, J. Kuhl, K. Ploog, P. Dawson, and C. T. Foxon, Phys. Rev. B 42, 5809 (1990).

${ }^{16}$ Y. Masumoto, T. Mishina, F. Sasaki, and M. Adachi, Phys. Rev. B 40, 8581 (1989).

${ }^{17}$ T. Itoh, Y. Iwabuchi, and M. Kataoka, Phys. Status Solidi B 145, 567 (1988).

${ }^{18}$ T. Itoh, Y. Iwabuchi, and T. Kirihara, Phys. Status Solidi B 146, 531 (1988).

${ }^{19}$ M. Ueta, H. Kanzaki, K. Kobayashi, Y. Toyozawa, and E. Hanamura, Excitonic Processes in Solids (Springer, Berlin, 1986), Chap. 3.

${ }^{20}$ S. Yano, T. Goto, S. Iwai, K. Edamatsu, and T. Itoh, Jpn. J. Appl. Phys. 34, Suppl. 34-1, 140 (1995).

${ }^{21}$ J. E. Eby, K. J. Teegarden, and D. B. Dutton, Phys. Rev. 116, 1099 (1959).

${ }^{22}$ T. Kawazoe and Y. Masumoto (unpublished). 